\title{
The New Ethical Responsibilities of Internet Service Providers
}

\author{
Luciano Floridi
}

Published online: 3 November 2011

(C) Springer-Verlag 2011

As the European Group on Ethics in Science and New Technologies and the UNESCO Observatory on the Information Society have documented, the exponential developments of internet services and resources have brought enormous benefits and opportunities to an increasing number of people. But it has also greatly outpaced our understanding of their conceptual nature and ethical implications, while raising unprecedented moral challenges, whose complexity and global dimensions are rapidly expanding, evolving and becoming increasingly serious. Examples come readily to mind. Consider the ethical issues arising from "the triple A", namely the availability, accessibility and accuracy of informational resources, independently of their format, kind and physical support; the corresponding questions concerning standards of reliability and trustworthiness of information sources; or e-inclusion and the so-called digital divide. The more we become accustomed to living and working immersed within an informational environment, the easier it becomes to unveil the new moral difficulties that we encounter. Thus, in recent years, information societies have been forced to deal with urgent, ethical problems concerning information privacy and confidentiality, hacking (understood as the unauthorised access to a computerised information system), digital vandalism (e.g. the creation and intentional dissemination of software viruses), security, monitoring and control (including issues related to digital warfare, terrorism and a dystopian "surveillance society"), freedom of expression, censorship, filtering and contents control. Likewise, the debate about information ownership and intellectual property (including copyright and patents legislation), fair use, piracy, and the development and support of open source software affects both users and producers ethically, while morally shaping their informational environment. Inevitably, similar issues constitute a complex and potentially confusing scenario, not least because it is in constant and rapid evolution. The lack of balance is obvious and a matter of daily experience in the life of millions of citizens.

\footnotetext{
L. Floridi $(\square)$

Department of Philosophy, University of Hertfordshire, de Havilland Campus, Hatfield, Hertfordshire AL10 9AB, UK

e-mail: 1.floridi@herts.ac.uk
} 
In this complex scenario, it is at least clear that internet service provider (ISPs) are major actors, whose decisions and actions significantly influence and shape the informational environment in which current and future generations will live and interact. The legal requirements that should be satisfied by ISPs are at the centre of a lively debate. Much less attention has been dedicated to their new ethical responsibilities. For this reason, the UNESCO Chair in Information and Computer Ethics, in collaboration with Google, organised a research workshop at the University of Hertfordshire, UK, in February 2011.

The task of the workshop was to fill that serious gap in our understanding, in order to see how ISPs might be able to equip themselves with a shareable and sustainable information ethics. The hope was that the workshop would contribute to anticipate difficulties, identify opportunities and resolve moral conflicts and dilemmas as far as ISPs' new ethical responsibilities are concerned. Some of the leading researchers in the area participated in the workshop: Dan Burk, Penny Duquenoy, Marco Gercke, Mireille Hildebrandt, Joe McNamee, Daniel Nagel, Sarah Oates, Ugo Pagallo, Wolter Pieters, Hendrick Speck, Matteo Turilli, Jeroen van den Hoven and Yorick Wilks. This issue collects a selection of their original contributions, revised and then assessed for publication. I am grateful to all participants for their timely effort in addressing such a pressing issue and for their feedback on the project and the following articles, and to Laura Bononcini, Deven Desai, Penny Driscoll, Nicklas Lundblad, and Marco Pancini for their indispensable support in organising the workshop. Vint Cerf, Google vice president, and Robert Madelin, director general for Information Society and Media of the European Commission, kindly accepted the invitation to share their views on the new responsibilities of ISPs. Their articles will be found in the commentary session. 